\title{
Jean-Guy Nadeau
}

Professeur, Faculté de théologie, Université de Montréal

(1987)

\section{"La gestion sociale et pastorale de la prostitution: une étude praxéologique”}

\begin{abstract}
Un document produit en version numérique par Jean-Marie Tremblay, bénévole, professeur de sociologie au Cégep de Chicoutimi Courriel: jean-marie_tremblay@uqac.ca

Site web pédagogique : http://www.uqac.ca/jmt-sociologue/
\end{abstract}

Dans le cadre de la collection: "Les classiques des sciences sociales"

Site web: http://classiques.uqac.ca/

Une collection développée en collaboration avec la Bibliothèque Paul-Émile-Boulet de l'Université du Québec à Chicoutimi Site web: http://bibliotheque.uqac.ca/ 
Cette édition électronique a été réalisée par Jean-Marie Tremblay, bénévole, professeur de sociologie au Cégep de Chicoutimi à partir de :

\section{Jean-Guy NADEAU,}

“La gestion sociale et pastorale de la prostitution: une étude praxéologique”.

Un article publié dans l'ouvrage sous la direction de Marcel Viau et Raymond Brodeur, Les études pastorales: une discipline scientifique ? Pastoral Studies as a Scientific Discipline ?, pp. 235-250. Québec : Université Laval, Groupe de recherche en sciences de la religion, 1987, 398 pp. Les Cahiers de recherches en sciences de la religion, vol. 8, 1987.

M. Jean-Guy Nadeau est professeur titulaire de sociologie des religion à la Faculté de théologie et de sciences religieuses de l’Université de Montréal.

[Autorisation formelle accordée par l'auteur le 5 octobre 2006 de diffuser cet article dans Les Classiques des sciences sociales.]

Courriel : Raymond.Lemieux@ftsr.ulaval.ca

Polices de caractères utilisée :

Pour le texte: Times New Roman, 14 points.

Pour les citations : Times New Roman, 12 points.

Pour les notes de bas de page : Times New Roman, 12 points.

Édition électronique réalisée avec le traitement de textes Microsoft Word 2004 pour Macintosh.

Mise en page sur papier format : LETTRE (US letter), 8.5'” x 11'’)

Édition numérique réalisée le 5 décembre 2006 à Chicoutimi, Ville de Saguenay, province de Québec, Canada. 


\section{Jean-Guy Nadeau}

professeur titulaire de sociologie de la religion et d'histoire du christianisme

à la Faculté de théologie et de sciences religieuses de l’Université Laval

\section{"La gestion sociale et pastorale de la prostitution: une étude praxéologique”}

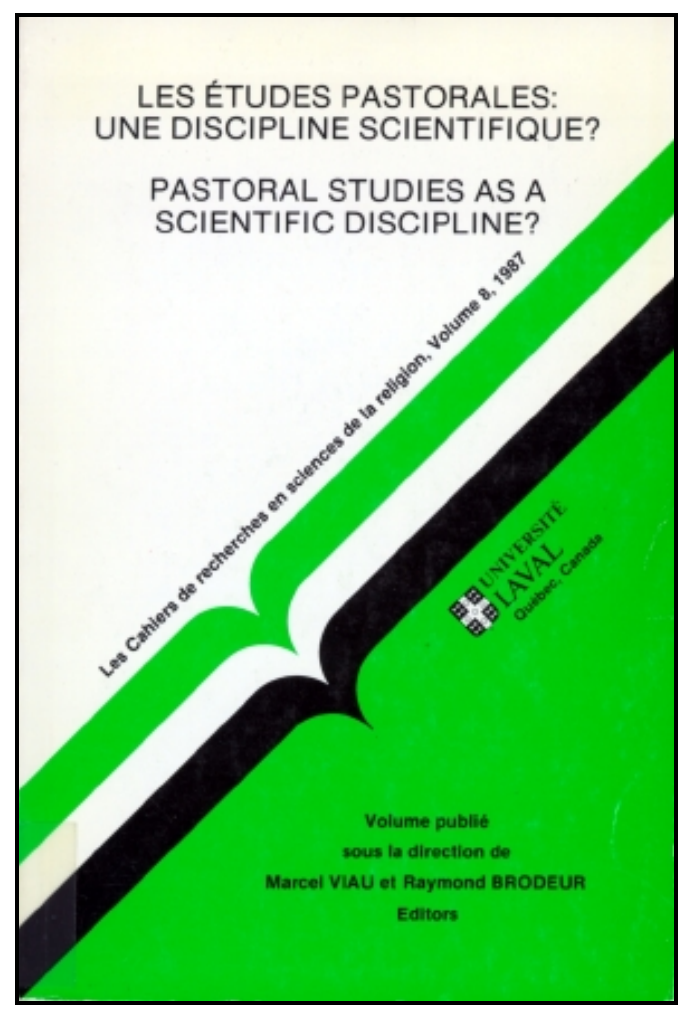

Un article publié dans l'ouvrage sous la direction de Marcel Viau et Raymond Brodeur, Les études pastorales: une discipline scientifique ? Pastoral Studies as a Scientific Discipline ?, pp. 235-250. Québec: Université Laval, Groupe de recherche en sciences de la religion, 1987, 398 pp. Les Cahiers de recherches en sciences de la religion, vol. 8, 1987. 


\section{Table des matières}

Introduction

1. La prostitution, une réalité complexe

2. La prostitution, une pratique symbolique déterminante

3. Un parcours praxéologique 
Jean-Guy Nadeau

“La gestion sociale et pastorale de la prostitution: une étude praxéologique”. *

Un article publié dans l'ouvrage sous la direction de Marcel Viau et Raymond Brodeur, Les études pastorales: une discipline scientifique ? Pastoral Studies as a Scientific Discipline ?, pp. 235-250. Québec : Université Laval, Groupe de recherche en sciences de la religion, 1987, 398 pp. Les Cahiers de recherches en sciences de la religion, vol. 8, 1987.

\section{Introduction}

$\underline{\text { Retour à la table des matières }}$

De 1971 à 1979, une travailleuse sociale ouvre son logement, rue Saint-Denis à Montréal, à des femmes qui vivent de la prostitution. Elles s'y rassemblent alors régulièrement avec un groupe de chrétiennes et de chrétiens intéressés à faire Église avec elles. Ce projet de pastorale informelle, que nous appellerons « Chez Marie », du nom de son animatrice, Marie Labrecque, se caractérisait par son souci de gratuité et de disponibilité, plutôt que de réhabilitation ou de conversion. Il aura permis à ses participants de vivre une expérience ecclésiale particulière que nous croyons significative aussi bien pour la tradition chrétienne que pour nos rapports sociaux.

Les femmes prostituées ont pu s'y rencontrer hors de leur occupation professionnelle, partager une expérience qui doit souvent se taire puisqu'elle n'est pas acceptée, rencontrer des gens qui ne prétendaient pas les utiliser, les juger ou les réformer. Certaines enfin ont pu y dé-

* Extrait de Entre le fantasme et la reconnaissance. Étude pastorale de pratiques de prostitution, thèse de Ph.D. (Théologie - Études pastorales), Université de Montréal, 1983, pp. 2-22. Nous présentons ce texte à titre d'illustration d'une problématique et d'un parcours de recherche en praxéologie pastorale. 
couvrir un visage de l'Église ou du Dieu de Jésus-Christ qui leur était jusqu'alors inconnu ou interdit. Leurs partenaires, d'autres participants ou des animateurs du projet, y découvraient une parole originale et provocante, prenaient conscience de certaines parentés avec des femmes qu'ils avaient cru étrangères et dont ils éprouvaient enfin l'importance pour le projet ecclésial comme pour l'imaginaire social.

Issue d'une participation de plus de cinq ans à cette pratique, notre étude en rend compte pour en dégager les enjeux concernant l'interaction socio-ecclésiale avec des personnes prostituées. Initialement, cette recherche universitaire visait à informer cette pratique dont elle apparaissait comme un moment critique. Conscients des limites et de la marginalité de leur projet, ses animateurs sentaient parfois le besoin d'en confirmer les options. Sa gratuité et sa discrétion étaient-elles justifiables relativement aux enjeux sociaux de la prostitution et du rapport aux femmes prostituées ? Le caractère privé et intime de l'accueil ne le rendait-il pas finalement illusoire ? Était-elle réaliste, cette prétention à révéler à des femmes prostituées qu'elles sont en réalité aimées de Dieu, alors qu'elles se trouvent marginalisées par leur Église ? Se posait enfin la question du partage eucharistique de cet amour, alors même que l'Assemblée des Évêques du Québec, qui se penchait sur les marginaux, les désignait encore comme des «nonsacramentalisables ».

Ces questions nous ont conduit à rechercher les assises sociopersonnelles et pastorales d'une pratique qui entendait se démarquer de l'ensemble des interventions (judiciaires, scientifiques, éducatives ou commerciales) relatives aux femmes prostituées. Une double pertinence, nous a-t-il semblé, demandait alors à être établie ou confirmée, tant du côté des prostituées et de leur vécu que du côté de la tradition chrétienne et de la mouvance du Royaume. Il s'agissait de dégager le rapport de la pratique et de ses discours avec la double quête portée, bien que peu affirmée, par ses "destinataires » et ses " animateurs ». Il s'agissait aussi de vérifier en quoi cette pratique, qui se voulait un espace d'ouverture dans l'enfermement des femmes prostituées, se démarquait des pratiques et des discours qu'elle soupçonnait de participer à cet enfermement. Il fallait donc prendre le temps d'étudier cette pratique, comme celles qu'elle contestait, de la situer en rapport à la tradition chrétienne, dont elle se réclamait, afin de pouvoir l'éclairer 
sur son interaction avec des prostituées, avec d'autres pratiques et avec le Règne de Dieu, pour proposer éventuellement une confirmation ou une transformation en profondeur de cette pratique.

«Parti » de la pratique que nous continuions de mener, nous y « revenions » donc après une démarche critique susceptible de la rendre davantage consciente de ses enjeux et de ses positions. On aura peut-être reconnu les préoccupations de la praxéologie pastorale élaborée à la Section des études pastorales de la Faculté de théologie de l'Université de Montréal : à partir de l'analyse des modes de la pratique, de son milieu, du discours de ses acteurs et de leurs interactions, dégager les modèles explicites ou implicites de cette pratique et essayer d'en assurer la cohérence, la pertinence et l'efficience, en rapport au pays réel et à la tradition chrétienne.

Le projet de «Chez Marie » s'est terminé avec le départ de son animatrice en 1979. Il a duré huit ans, ce qui est relativement long pour une intervention en milieu marginal, privée et libre d'attaches ou de supports institutionnels. La prospective de la recherche qui l'avait accompagnée jusque là s'en trouvait donc modifiée. Néanmoins, les questions qu'elle portait demeuraient pertinentes étant donné leur caractère d'universalité et leur importance fondamentale ${ }^{1}$. Sa dynamique et certains des discours qu'elle avait rencontrés menaient en effet à critiquer la privatisation massive de la problématique prostitutionnelle aux niveaux politique, scientifique et ecclésial, et sa réduction encore trop fréquente à un seul de ses acteurs : la femme prostituée. La réalité prostitutionnelle s'y révélait comme un phénomène beaucoup plus large, dépassant les plans commercial et moral auxquels on la limite souvent, et qualifiant même un certain nombre de comportements interpersonnels, collectifs et ecclésiaux sur lesquels nous étions amené à réfléchir.

1 Le fait que la recherche se poursuive en ce sens témoigne de la portée de la pratique de « Chez Marie », dont elle est issue. 


\section{La prostitution, une réalité complexe}

La prostitution constitue un phénomène connu et relativement important dans notre société. La conjoncture actuelle, dans la mouvance du Comité de Protection de la Jeunesse et d'un groupe d'intervention affilié au Bureau Consultation Jeunesse de Montréal, attire davantage l'attention sur la prostitution des garçons qui a connu ici une croissance rapide depuis dix ans. La prostitution des femmes ou des jeunes filles reste cependant le "modèle " fondamental de toute prostitution ou du discours sur la prostitution. Elle constitue, dans notre culture, le phénomène prostitutionnel le plus important, aussi bien structurellement que quantitativement. Au Canada, par exemple, la question est régulièrement portée en Cour suprême depuis quelques années, et les corps policiers ne cessent de presser le parlement fédéral de leur accorder les moyens de réprimer ou de contrôler le trafic prostitutionnel. La loi est cependant liée au consensus social et celui-ci, tout en restant divisé, semble évoluer avec la libéralisation des moeurs sexuelles et la problématique des droits individuels. Ainsi la Cour suprême a-t-elle considérablement resserré la définition de la sollicitation ou du racolage. En octobre 1982, un juge de Toronto cassait un jugement contre une jeune femme accusée de sollicitation, soulignant entre autres motifs qu'on n'inculpe pas les politiciens qui interpellent les passants pour quémander leur vote. Par ailleurs, plusieurs municipalités, dont Montréal, ont légiféré pour interdire aux "prostituées » de se tenir dans des endroits publics ; mais leurs règlements ont été contestés par des groupes de citoyens et portés devant la Cour suprême.

Malgré l'acceptation de la prostitution qui se profile en fait la prostitution ne constitue pas en soi un délit au Canada, la prostituée demeure une figure importante de notre imaginaire culturel : la prolifération des rôles de prostituées au cinéma témoigne de ce double fait. Le 
vocabulaire désignant la femme qui vit de la prostitution fait partie de nos moeurs et du langage commun : prostituée, "putain ", fille de gaffe, " guidoune », pécheresse ${ }^{2}$. Or, ce vocabulaire favorise l'identification de la prostituée à une activité féminine. On notera plus loin la pauvreté et la généralité du vocabulaire qui désigne l'homme en prostitution, que ce soit à titre de prostitué ( " gigolo » ou « serin »), ou de " client», terme bien général et finalement assez noble. Parler sans plus de prostituée ou de prostitution féminine, c'est laisser entendre que la femme est seule en cause dans cette affaire. En réalité, elle y serait plutôt minoritaire si l'on songe au nombre d'hommes qui l'y déterminent ou y participent avec elle : clients, souteneurs, journalistes, spécialistes des sciences humaines, etc. En fin de compte, cette prostitution, même si elle engage la femme qui s'en trouve sur-déterminée ou stigmatisée, n'est pas seulement « féminine », mais d'abord hétérosexuelle.

On se croira peut-être loin des préoccupations théologiques ou pastorales. Pourtant, les clercs aussi font partie de ces hommes qui, par leur discours et leur pratique, ont déterminé le corps des femmes prostituées. La morale chrétienne, particulièrement sensible à la gestion ou à la maîtrise de la sexualité humaine, s'est, dès ses débuts, prononcée sur la prostitution, influençant ou justifiant souvent le législateur civil. De même, la pastorale de la prostitution, ou plus précisément la pastorale des prostituées, connaît une longue histoire qu'on fait remonter à la pratique même de Jésus. Marie-Madeleine est une des figures évangéliques les plus connues! Aujourd'hui encore, la pastorale se préoccupe des prostituées et, lorsqu'en juin 1975, les prostituées françaises ont voulu se faire entendre, c'est à l'église qu'elles sont allées... et qu'elles ont été le mieux reçues.

Enfin, la femme prostituée constitue une figure clef de l'Ancien Testament et l'une de celles qui importent au Nouveau Testament. Le thème de la prostitution d'Israël se retrouve aussi dans l'histoire de l'Église que certains, selon leurs positions politiques ecclésiales, considèrent comme une prostituée rachetée ou abandonnée par son

2 On aurait cru ce terme inactuel, or on le retrouve dans les titres d'articles portant sur la fronde des prostituées françaises en 1975 ! 
Seigneur. C'est indiquer le caractère et l'importance symboliques de la prostitution, tant en termes métaphoriques qu'en termes relationnels.

\section{La prostitution, une pratique symbolique déterminante}

$\underline{\text { Retour à la table des matières }}$

L'observateur attentif finit par découvrir que la prostitution implique des rapports plus complexes qu'il n'y paraît à première vue. La prostituée et le client, voire le souteneur, ne sont pas seuls en cause. Un large milieu socio-économique et culturel soutient leur relation, l'organise, la promeut ou la contrôle. Cette pratique, que l'on croit privée, est d'abord sociale. Si l'on reconnaît que la relation sexuelle ne met jamais en relation que des individus, mais aussi la culture et le langage qui les portent, on saisira la détermination de ces facteurs pour la relation prostitutionnelle. Des rapports multiples la précèdent et la structurent, alors que différents acteurs, plus ou moins occultés par leur pratique ou le discours social, l'exploitent à divers niveaux : économique, affectif et politique, mais aussi intellectuel et moral.

La prostitution apparaît comme une tactique dans la quête humaine de reconnaissance et d'identité, particulièrement lorsqu'elle s'inscrit en rapport à la misère sexuelle et la dépendance économique. La prostitution est en ce sens une activité symbolique dont le plaisir sexuel constitue évidemment un élément majeur, et non pas seulement une activité commerciale ou sexuelle, voire "hygiénique », comme on le prétend parfois. À ce titre, la prostitution nous « fait » ou nous identifie, comme elle fait la prostituée, le souteneur, le client, le policier de " son » escouade, le journaliste à potins, l'expert, etc. Mais elle ne nous fait pas qu'individuellement. Son interaction symbolique est aussi collective, comme le confirment des éléments des sciences humaines et de la tradition chrétienne. 
La psychologie et la psychanalyse dévoilent cette quête comme le drame de la prostituée, du client et du souteneur. La sociologie, surtout l'interactionnisme symbolique, indique que c'est aussi le drame de l'ensemble social. Le discours théologique révèle qu'il se joue là un drame pour tout humain en relation à Dieu.

Ce qui spécifie la prostitution de l'autre puisque prostituer est d'abord un verbe transitif comme tactique d'identification, c'est qu'elle conjugue la négation de l'autre, et l'exploitation de sa disponibilité ou de sa quête de reconnaissance, avec les signes de la communication. La prostitution opère comme simulacre de rencontre et consommation de relation. C'est l'anti-relation qui joue à la relation, l'« anti-verbe » 3 ou le scénario qui simule la parole et la rencontre, échappant ainsi au risque de la reconnaissance de l'Autre. La parole de l'autre y devient une extension du Même, une scène où le « prostituant » peut incarner ses fantasmes, s'identifier vis-à-vis soi et vis-à-vis l'autre ainsi réduit.

La prostitution apparaît alors très proche du viol, comme l'indiquent les féministes qui voient dans ces phénomènes et ces pratiques le coeur de la condition sociale de la femme ${ }^{4}$. La prostitution se démarque cependant du viol : le sujet prostitué consent à la situation dont il tire un certain profit, même s'il s'y trouve piégé, personnellement et socialement marqué. La société exonère l'homme client mais stigmatise la femme prostituée, la réduit à un rôle où elle l'enferme : « elle n'est qu'une putain ».

Si le client s'assure par contrat tacite de la disponibilité de la prostituée, il en va autrement de la pratique du corps social. Celui-ci ravit en effet cette disponibilité et impose à la prostituée un scénario qu'elle est obligée de subir, comme dans la prostitution la plus contrôlée ou l'« abattage » (prostitution à la chaîne). L'analyse sociologique dévoile une société qui prostitue la femme prostituée, redondance du langage

3 L'expression est de Michel M. Campbell, « Rosemary's Baby ou Anti-Verbe fait chair » : Sciences Religieuses / Studies in Religion, 1/4 (1972), 269-290.

4 ans sa préface à l'étude de Suzan Brownmiller, Le viol, Benoîte Groult situe " le viol au coeur de la condition féminine » (p. 14) ; dans sa préface à La Dérobade de Jeanne Cordelier, c'est la prostitution qu'elle situe « au coeur même de la condition féminine » (p. vi). 
qui indique la violence et la «duplicité » de ce processus social d'enfermement et d'exploitation.

L'exploration critique des discours des sciences humaines et de la tradition chrétienne sur la prostitution découvre que ce geste d'enfermement y est largement repris. Largement, mais non pas absolument, puisque la tradition scientifique comme la tradition chrétienne sont plurielles, recelant aussi des discours et des pratiques qui dénoncent l'enfermement et promeuvent même la reconnaissance des personnes prostituées. Ces discours demeurent cependant minoritaires, alors que les discours dominants s'inscrivent dans une matrice prostitutionnelle où la quête de reconnaissance et d'identité est flouée et enfermée dans un rituel de consommation socialement contrôlé et exploité.

Ce n'est donc pas seulement dans le lit ou au bordel que l'autre est prostitué et que nous le sommes avec lui, puisque J'activité est symbolique -, mais bien à chaque fois qu'on exploite la quête de l'autre en jouant à la relation dans une situation d'anti-relation, chaque fois qu'on réduit l'autre à ses signes et qu'on l'y enferme pour se rassurer sur sa propre identité. C'est en ce sens que la prostitution est saisie comme l'archétype du rapport phallocrate ou «machiste » au corps de la femme.

On nous a souvent demandé ce qu'un homme faisait encore à parler de prostitution féminine plutôt que de prostitution masculine. N'étaitce pas, à nouveau, usurper une parole qui n'était pas la sienne, se braquer sur la femme et parler d'elle, voire parler pour elle ?

Dans un premier temps, on aurait pu penser que la recherche, n'échappant pas à un conditionnement socio-culturel important, allait en ce sens. La pratique dont elle émergeait ne se centrait-elle pas effectivement sur les femmes prostituées ? Cette pratique, cependant, s'affirmait comme une pratique de femmes où les hommes étaient très minoritaires, et son discours s'avérait fort critique envers le rôle des hommes dans la prostitution : policiers, clients, père, ami... La parole des prostituées pouvait s'y déployer sans entraves, dévoilant l'interaction masculine à la prostituée. Très tôt, les femmes prostituées nous ont sensibilisé au statut de client ou d'exploiteur potentiel auquel se réduit tout homme à leurs yeux. Parler de prostitution féminine, ou en 
faire un problème exclusivement féminin, s'avère désormais un processus d'occultation du rôle de l'homme dans la prostitution.

Homme, c'est précisément ce rôle que nous voulons mettre en lumière. Notre étude ne vise pas tant la femme en prostitution, cette dimension relevant davantage d'une parole féminine, que l'homme en prostitution. Elle tend donc à focaliser sur l'interaction prostitutionnelle une attention le plus souvent dirigée sur la femme prostituée. Défi de taille, étant donné nos conditions sociales et culturelles.

Nous analysons cette interaction selon un triple rapport. D'abord celui du bordel, où le rapport prostitutionnel s'énonce clairement. La problématique qu'en dégagera l'analyse servira à comprendre les dynamiques des autres pratiques de prostitution. Le second rapport est celui de la concentration des interventions et des discours socioecclésiaux sur la femme prostituée. Le troisième est le rapport métonymique symbolisé par la prostitution. Cette triple pratique est particulièrement révélatrice de la condition masculine et de sa gestion des rapports humains. D'où l'intérêt d'une parole masculine à l'écoute de celle des femmes et (auto)-critique par rapport à l'interaction prostitutionnelle.

Signalons enfin que le dévoilement métaphorique de la prostitution dépasse largement le rapport hommes-femmes. La tradition socialiste a stigmatisé comme prostitution la situation de l'ouvrier industriel. L'Ancien Testament symbolise par cette relation l'infidélité d'Israël à son Seigneur, et l'Église de Jésus-Christ elle-même s'est parfois trouvée parfois identifiée à une prostituée.

La prostitution parle donc de nous, de notre rapport à l'autre, à la parole, et même à Dieu. En mettant en évidence les aspects collusoires, consommatoires et politiques de ces rapports, nous en faisons un lieu privilégié pour saisir les modes et les enjeux de ces relations qui se trouvent aussi au coeur du drame ecclésial. C'est finalement là l'enjeu même de notre étude, son projet. Interpellé par une pratique particulière, nous avons choisi de l'analyser et de la comprendre par la voie praxéologique. Reste à établir les articulations de cette voie, en rapport à notre propre démarche. 


\section{Un parcours praxéologique}

\section{$\underline{\text { Retour à la table des matières }}$}

La démarche de praxéologie pastorale est en élaboration depuis dix ans à la Section des études pastorales de la Faculté de théologie de l'Université de Montréal ${ }^{5}$. Praxéologique, elle a pour visée de faire émerger le discours d'une pratique et de le confronter à ses porteurs et à ses référents, pour rendre la pratique davantage consciente de ses enjeux, modes et langages, et accroître son efficience. Pastorale, elle vise des pratiques qui, d'une façon plus ou moins explicite, se fondent sur la reconnaissance de Jésus-Christ. Elle accorde donc à la tradition chrétienne une place de choix parmi ses référents critiques. On trouve dans la praxéologie pastorale le cadre méthodologique et les principaux éléments de notre étude : observation d'une pratique et émergence des discours et des modèles de ses acteurs ; confrontation de ces modèles à l'environnement de la pratique et à d'autres discours ou pratiques scientifiques, politiques, ecclésiaux, bibliques, etc. ; saisie de leurs enjeux et de leurs implications pour la pratique. Ainsi renvoyée à la cohérence, à la pertinence et à l'efficience de sa gestion des interrelations entre ces facteurs, la pratique est souvent appelée à se ressaisir (dans ses modèles ou dans ses objectifs, par exemple) ou à se modifier.

On reconnaît quatre opérations majeures dans cette démarche : observation, interprétation, intervention, prospective. Si cette exposition paraît linéaire, son processus s'avère en fait une réelle dialectique entre ces opérations, elles-mêmes déterminées par diverses instances critiques. Signalons des instances d'ordre cognitif (du côté de l'analyse stratégique et de l'herméneutique), des instances d'ordre psycho-social

5 Voir les cahiers Études Pastorales (1971 à 1976), Université de Montréal, Faculté de théologie, et le Fichier-cadre de Dossier recherche-action en praxéologie pastorale, Première partie: Questionnaire; Deuxième partie: Textes d'appoint. Université de Montréal, Faculté de théologie, 1977. 
(du côté du praxéologue et des acteurs de la pratique), et enfin des instances de l'ordre de la spécificité pastorale, lorsque les agents de la pratique ou de la recherche s'en réclament.

La prospective, par exemple, concerne aussi bien la première articulation des souvenirs et des rêves portés par la pratique dont ses options de foi, si elle est pastorale, que la prévision des effets et des tâches de cette pratique. Elle sous-tend ainsi l'ensemble de la démarche, comme elle porte déjà l'ensemble de la pratique. Elle ne saurait donc être confinée en fin de parcours, et on la retrouve dans la perspective de l'observation, dans les choix de l'interprétation, dans les objectifs fondamentaux de l'opérationnalisation de l'intervention, etc.

Quant à l'interprétation théologique, elle émerge déjà dans l'analyse de la pratique pastorale dont elle constitue un moment clef, alors que la quête de ses référents s'articule d'abord comme une observation du champ des sciences humaines et de la tradition chrétienne. Il en va ainsi pour chacune des opérations praxéologiques, en interrelation étroite avec les autres qui la déterminent et qu'elle détermine aussi. Indiquons leur mouvement à travers notre recherche.

Observer une pratique ${ }^{6}$, c'est d'abord prendre conscience de ses modes, de ses objectifs, de ses résultats et de ses tendances. C'est en même temps être à l'écoute de ses acteurs : leurs discours, leurs interactions, leurs histoires et leurs rêves. S'il s'agit d'une pratique d'intervention sociale ou d'une pratique pastorale, l'observation se fera attentive aux liens entre l'acte de proposition d'un groupe d'animateurs, acte auquel on limite trop souvent le compte rendu des objectifs d'une pratique, et la quête, ou la demande, du groupe « client », lui aussi sujetporteur de la pratique.

Observer une pratique, c'est aussi la situer dans le milieu géophysique et socio-culturel qui la détermine et avec lequel elle est en interrelation, ne serait-ce que par les intérêts divers de ses acteurs. Il s'agit donc d'analyser aussi le champ des pratiques sociales et ecclé-

6 Nous sommes redevables, pour ces réflexions, à nos collègues du Séminaire clinique d'intervention pastorale de la Faculté de théologie : voir les procèsverbaux et documents à la Section des études pastorales de la Faculté. 
siales dans lequel s'insère la pratique pastorale en cause. Une pratique d'intervention entend en effet répondre à des exigences ou à des drames du milieu. Or, il est probable que d'autres acteurs du milieu soient aussi sensibles à ces drames et tentent de les gérer par le moyen d'autres pratiques dont l'étude praxéologique doit aussi tenir compte.

Ce type d'attention au vécu de «Chez Marie » et aux préoccupations de ses acteurs issus d'horizons variés de la rue Saint-Laurent et à la Faculté de théologie, en passant par le discours des sciences humaines caractérise notre recherche. Il nous a permis de découvrir une dramatique commune à ces lieux divers et de la mettre en relief.

Notre premier chapitre étudie donc la pratique de "Chez Marie » : d'abord son vécu quotidien et ses interactions personnelles et collectives ; puis l'histoire et la parole de ses acteurs, femmes prostituées et, enfin, intervenants. L'observation ne s'appuyait pas au départ sur une hypothèse qu'il eût fallu vérifier, mais sur une volonté d'immersion dans une pratique qui nous précédait et dont nous entendions découvrir, respecter et approfondir la spécificité. La pratique se déployait dans l'axe d'une rencontre gratuite avec des femmes prostituées socialement situées tout à l'opposé de ce souci. Les premiers éléments de nos modèles d'analyse et le soupçon des enjeux que dévoile notre étude ont d'abord émergé de cette rencontre de perspectives relativement contraires.

C'est dire que " Chez Marie » renvoie à des mondes et à des pratiques diverses dont elle prétend se démarquer ou s'inspirer : pratiques prostitutionnelles elles-mêmes, discours des sciences humaines, pratiques ecclésiales, pratiques évangéliques. L'étude de ces pratiques, à laquelle nous convie la démarche praxéologique, révélera des enjeux communs aux rapports privés et collectifs qui s'y inscrivent et qui semblent fondamentaux pour l'Église comme pour la société.

Le second chapitre explore la pratique de la prostitution qui constitue un référent constant à la pratique de "Chez Marie ». D'une part, en ce que ce projet est né en réaction à la marginalisation des femmes prostituées ; d'autre part, en ce que la pratique prostitutionnelle détermine profondément la parole de ces femmes. 
Ce chapitre se déploie en quatre temps. Il analyse d'abord les modes de la rencontre prostitutionnelle qu'il regroupe en trois séquences : sociale, monétaire, sexuelle. Il examine ensuite les discours des acteurs prostituées, clients, policiers à l'intérieur de la rencontre ellemême. Vient alors un premier recours aux sciences humaines pour élaborer, en confrontation avec les analyses précédentes, une première problématique de la prostitution en tant que relation de consommation fétichiste, relation de ritualisation de la violence, relation de domination fantasmatique de l'autre. Nous définissons enfin la prostitution comme une quête de reconnaissance flouée, bien que relativement opérante, ou mal-opérante.

Les sources littéraires et scientifiques, comme l'indique un vocabulaire plus technique, se conjuguent ici avec l'observation du terrain, permettant de prendre une meilleure distanciation et d'assurer le regard. Elles deviendront les matériaux privilégiés des prochains chapitres qui s'intéressent aux discours critiques sur la prostitution, discours scientifiques et théologiques qui marquent aussi la pratique de " Chez Marie » et dont seule une large revue de littérature permettait l'étude.

Les sciences humaines et la tradition chrétienne, qu'on utilise le plus souvent comme référents exclusifs de sens, se révèlent aussi, et même d'abord, des lieux de pratiques, des lieux d'options, ce qu'occultent souvent leurs discours qui se donnent des airs d'évidence que la praxéologie ne saurait simplement entériner. En ce cas, ce sont leurs pratiques d'interaction avec des femmes prostituées qui ont retenu notre attention. Une des originalités de notre démarche est de s'y intéresser à ce titre et de confronter leurs modèles de compréhension aux modèles tirés d'une pratique singulière, pour en dégager une matrice qui rende compte d'une expérience plus universelle.

Le troisième chapitre fait l'histoire des discours des sciences humaines sur la prostitution. Des approches biosomatiques et psychologiques centrées sur la femme prostituée, il va aux approches fonctionnalistes et critiques davantage attentives à l'institution prostitutionnelle, puis à celles qui dégagent l'interaction sociale avec la prostituée. Il étudie enfin le discours féministe en tant que parole en interaction. Le dernier temps de ce chapitre dévoile les mécanismes prostitutionnels dominants de même que les quelques effets d'ouverture critique à 
l'oeuvre dans ces discours et dans leurs pratiques. La prostitution et ses discours critiques apparaissent alors comme une donnée essentielle des rapports entre hommes et femmes déjà au coeur des précédents chapitres. Le chapitre se termine sur l'interpellation des spécialistes des sciences humaines, massivement masculins, dans leur rapport actif à la prostitution de la femme, et il invite à une parole qui ne s'occulte plus dans l'objectivation de l'autre, féminin.

L'observation et l'interprétation pastorales ne sauraient évidemment se limiter à l'étude du pays réel et des discours scientifiques. Émergeant déjà dans l'observation de «Chez Marie » et colorant notre problématique de la prostitution, l'interprétation théologico-pastorale détermine aussi un temps spécifique de la démarche. Il importe alors de dégager, du côté de la tradition chrétienne, les référents propres à la pratique étudiée.

Comme dans le cas des discours des sciences humaines, nous nous intéressons à l'histoire ecclésiale et à l'Écriture Sainte, non seulement pour les lumières qu'elles apportent à la compréhension de nos pratiques, mais parce qu'elles sont elles-mêmes constituées de pratiques dont certaines marquent le rapport à la prostitution et aux femmes prostituées.

Bien que la référence à l'Écriture Sainte paraisse plus déterminante pour la pratique de "Chez Marie ", où elle est néanmoins fort discrète, c'est par l'étude des rapports historiques de l'Église à la prostitution que nous abordons la suite de notre étude. Deux facteurs motivent ce choix.

La réflexion pastorale est souvent portée à court-circuiter les longs cheminements de l'histoire, fût-elle ecclésiale, pour retrouver la pratique de Jésus et s'y référer d'une façon plus ou moins mythique ${ }^{7}$. Le discours théologico-pastoral fonctionne alors comme si tout était déjà dit et qu'il ne s'agissait plus que de faire oeuvre d'intelligence (inspi-

7 C'est un peu ce qui se passe lorsque la réflexion théologique sur la sexualité humaine débute avec Genèse 1 et 2, ou s'y braque, oubliant même la suite de l'Écriture, particulièrement ses textes narratifs où apparaissent les pratiques historiques de la sexualité judéo-chrétienne. 
rée) pour l'expliquer, ou de bonne foi pour le comprendre. Or l'histoire, déjà constitutive de l'Écriture, est aussi déterminante pour la pratique pastorale et la réflexion théologique.

Le second motif va dans le même sens. Notre lecture de l'Écriture est largement modulée, voire déterminée par nos pratiques et nos sensibilités, certes, mais aussi par celles de l'histoire qui nous a faits et par une problématique contemporaine qui la porte aussi. Sans faire l'herméneutique de cette problématique, il importe tout de même d'indiquer, ne fût-ce que structurellement, la distance historique entre nos pratiques et l'Écriture.

Le quatrième chapitre fait donc l'historique du rapport ecclésial à la prostitution. Dès ses débuts, l'Église s'est intéressée au sort des femmes prostituées et à leur conversion, sa pastorale se démarquant alors des pratiques socio-culturelles de son temps. Cet intérêt ne s'est pas démenti au cours des siècles, bien qu'il ait pu, ultimement, accroître la marginalisation et la stigmatisation des femmes prostituées. Par ailleurs, son discours moral qui a longtemps toléré la prostitution comme un mal nécessaire, a connu une large audience auprès du législateur, marquant et justifiant le rapport socio-politique à la femme prostituée. Ce discours a toutefois fortement évolué. Marqué par le souci de la dignité de la personne, il s'accorde aujourd'hui avec une pastorale plus attentive à l'autre. C'est cette évolution que nous voulons marquer avant d'en dégager la tension entre l'exclusion et l'accueil, tension déterminante pour l'édification de l'Église.

L'Église reconnaît un rôle fondamental pour sa pratique et son discours à l'événement Jésus-Christ consigné dans le Nouveau Testament. Or cet événement s'inscrit dans la dynamique d'alliance entre le Seigneur et Israël, mise en scène et interprétée dans l'Ancien Testament.

Notre cinquième chapitre étudie donc le rapport scripturaire à la prostitution. Il importe d'abord de saisir l'interaction de l'Ancien Testament à la prostitution qui s'avère un des pivots symboliques de la théologie de l'alliance. Qu'il s'agisse de prostitution profane, sacrée ou métaphorique, les enjeux vétéro-testamentaires de la prostitution sont ceux mêmes de l'alliance : l'identité et la reconnaissance du peuple élu 
et de son Seigneur. Le fait est net en ce qui regarde la prostitution sacrée et l'usage métaphorique de l'image de la prostituée désignant l'infidélité d'Israël. Nous aurons à l'établir dans le cas de la prostitution profane qui menace la légitimité de la descendance patrilinéaire.

Signalons que dans l'un ou l'autre cas, c'est toujours la femme ou la figure de la femme qui est stigmatisée dans le discours vétérotestamentaire. Pour être normal dans une société patriarcale, le fait n'en reste pas moins important pour la lecture que nous faisons aujourd'hui de ces textes.

La figure de la prostituée dans l'Ancien Testament est importante pour la compréhension de la pratique de Jésus relativement aux femmes prostituées. Leur préséance dans le Royaume continue de poser question, tout en étant largement rappelée par les pratiques pastorales d'aujourd'hui. Mais la figure néo-testamentaire de la prostituée ne se limite pas à ses relations avec Jésus de Nazareth. l'Église apostolique dessine un tout autre profil de la prostituée, qu'elle range du côté du diable et de la mort. Il faudra donc éclairer ce double rapport et ses enjeux pour l'identité chrétienne.

Le sixième chapitre s'élabore comme un temps de synthèse et de prospective théologiques et pastorales. Il rappelle les modèles de relations aux femmes prostituées qui ont été dégagés aux moments précédents, et il en établit la commune dramatique que l'interprétation a dévoilée dans la rencontre prostitutionnelle de "Chez Marie », dans les pratiques des sciences humaines, dans l'alliance biblique et la reconnaissance du Dieu de Jésus-Christ. Un premier type de rapport, majoritaire et occultant ses propres conditions de production, réduit la prostitution à l'activité de la femme et celle-ci, à un objet dont il dénie la parole. Un second, sensible à l'interaction, s'élabore comme une rencontre de l'autre dont il assume l'inconnu et les risques. On désignera le premier type comme un modèle d'enfermement, "prostituant ", et le second comme un modèle de croissance, "reconnaissant » ou convivial.

Ces modèles, dont nous avons indiqué les différents niveaux individuel et collectif, forment les pôles d'une même matrice qui permet de les intégrer et de reconnaître leur enjeu fondamental : la reconnais- 
sance et l'identification de soi dans le rapport à l'autre, et particulièrement à l'autre féminin, puisque la stigmatisation prostitutionnelle opère en ce sens.

Or l'Église s'identifie justement en fonction de cette double quête de reconnaissance et d'identité dont elle assure avoir la clef. Le salut dont elle est gardienne, témoin, levain, etc. selon les images qu'elle se donne d'elle-même, concerne aussi bien les relations entre les hommes... et les femmes, que les relations avec Dieu.

Notre démarche et la critique praxéologique nous amènent alors à demander comment l'Église gère cette quête et comment sa pratique de celle-ci l'identifie. Est-ce comme un sacrement de rencontre où la parole de l'autre est reconnue, et la quête vécue comme une élaboration commune sujette au risque du refus et de la transformation de soimême ? Est-ce comme un simulacre prostitutionnel où la parole est enfermée à l'avance dans un scénario sûr, et où l'Autre ainsi que la communauté de la quête sont déniés et exploités dans la consommation des signes de la relation ? Bien que ces pratiques ne soient probablement pas aussi tranchées dans la réalité, leur modellisation permet de dégager leur enjeu radical : l'identité ecclésiale même et sa propre quête. C'est en fonction d'elles que nous poserons alors la question du rapport à l'Autre et à l'autre prostitué, dont la pratique et le discours du Seigneur soulignent l'importance pour l'Église.

Puisque ce sont nos interactions avec l'autre qui nous identifient, notre croissance ou notre enfermement communs dépendent de ces rapports. La question de la place et du rôle des femmes, et des femmes prostituées, dans le corps ecclésial se dessine donc sur cet enjeu fondamental : rester enfermés avec elles dans une pratique qui les fantasme comme "putains »; croître avec elles dans une pratique qui prend le risque de les reconnaître comme " femmes » et " soeurs ", et dont " Chez Marie » peut apparaître comme un paradigme, tout juste ébauché, mais interpellant.

Fin du texte 